\title{
MACRO+: A Network Coding Driven Integrated MAC/Routing Protocol for Multihop Wireless Networks Invited Paper
}

\author{
L. Galluccio, A. Leonardi, G. Morabito, S. Palazzo \\ DIIT, University of Catania, Italy \\ email: \{name.surname\}@diit.unict.it
}

\begin{abstract}
Most next generation mobile terminals will be equipped with location computation facilities, e.g., GPS cards, which can be extremely useful to the purpose of routing in mobile multihop wireless networks. In fact, if nodes are aware of their own location, then geographical routing algorithms can be applied which do not require signaling and thus, are very efficient in high mobility scenarios. In this context, MACRO is an integrated MAC/routing protocol that achieves high energy efficiency in wireless multihop communications. The basic concept underlying MACRO is that when a node forwards a data packet, all other nodes in the radio coverage evaluate their goodness in forwarding the packet towards the destination. The best a node is to this purpose, the sooner it will try to take the responsibility of relaying the packet. The basic concepts of MACRO are revised in this paper to allow the exploitation of network coding which can further improve energy efficiency. The resulting routing scheme, called MACRO+, is assessed through analysis.
\end{abstract}

\section{Categories and Subject Descriptors}

C.2 [Computer-communication networks]: Network Protocols.

\section{General Terms}

Algorithms, Performance .

\section{Keywords}

Location aware protocols, geographical routing, network coding.

\section{INTRODUCTION}

The ever increasing diffusion of sophisticated wireless communication terminals and their announced support of open OSs will enable effective services based on the peer-to-peer

Permission to make digital or hard copies of all or part of this work for personal or classroom use is granted without fee provided that copies are not made or distributed for profit or commercial advantage and that copies bear this notice and the full citation on the first page. To copy otherwise, to republish, to post on servers or to redistribute to lists, requires prior specific permission and/or a fee. MOBILWARE 2008, February 13-15, Innsbruck, Austria

Copyright $\odot 2008$ ICST 978-1-59593-984-5

DOI 10.4108/ICST.MOBILWARE2008.2891 multihop communication paradigm [2], also known as ad hoc networking paradigm.

Energy efficient routing is crucial to the success of such services [13]. In this context, the fact that most next generation handheld communication terminals will be equipped with position calculation facilities, e.g., GPS cards, makes it possible the deployment of geographical routing algorithms. Such algorithms only require that terminals are aware of their own position and the position of the final destination. Accordingly, no signaling is required for routing purposes, which is extremely convenient in the case of high terminal mobility. As a consequence, geographical routing has been the focus of a large research effort in the last few years $[17$, $10,14]$.

In [4], we have introduced an integrated MAC/routing scheme for geographical routing called MACRO. This solution was tailored to wireless sensor networks, nevertheless the proposed approach can be applied to any wireless multihop communication scenario in which energy is a scarce resource. The basic idea of MACRO is that when a relay transmits a packet, all other nodes in its radio coverage will evaluate their goodness in forwarding the packet towards the destination. The better the position of a node is, the earlier it will try to take the responsibility of forwarding the packet. A similar idea was previously proposed in [17]; however MACRO also considers the case in which a node can select the most appropriate transmission power level.

In the last few years network coding has been proposed for wireless ad hoc networks [1, 3, 7], and a large research effort has been devoted to this subject. In fact applying network coding, the amount of information transferred at each transmission increases and therefore, energy consumption decreases.

However, at the best of our knowledge the combination of network coding and geographical forwarding has not been already studied. In this paper we revise the basic concepts of MACRO to take into account the possibility of applying network coding. We call the resulting scheme MACRO+. According to MACRO+, when a node listens a packet transmission it evaluates its goodness to forward such packet towards the destination, like in MACRO. It also evaluates its goodness to forward such packet combined, according to linear network coding, with one of the other packets that have been received recently and have not been forwarded yet. The higher the goodness, the sooner the node will try to take the responsibility of forwarding the packet. Evaluation of the above goodness is not trivial and a methodology has 
been derived in this paper. We demonstrate the advantages of applying network coding along with geographical routing through numerical examples.

The rest of the paper is organized as follows. In Section 2 we report the required background on geographical routing and network coding. In the same section we also report the basics of MACRO. In Section 3 we introduce MACRO+ which will be evaluated through numerical examples in Section 4. Finally, some conclusions will be drawn in Section 5 .

\section{RELATED WORK}

In these sections we revise some related work in the fields of geographical routing and network coding.

\subsection{Geographical routing}

Geographic forwarding [10] is proposed as a methodology for forwarding data from a sender to a destination, progressively reducing the distance between the source and the destination. A local optimal choice is performed where each relay node chooses as next hop the best neighbor in its proximity, i.e., the node in its neighborhood geographically closest to the destination. The greedy choices are thus performed based only on the local information about nodes' neighbors. In case holes are met in the network and, thus, greedy forwarding cannot be applied, perimeter forwarding can be invoked.

In [14] a geographical routing protocol working also in case of absence of location information is proposed. This protocol assumes that nodes do not know their GPS location but use a sort of virtual coordinates. This allows them to overcome the problem of not possessing location information. In [15] the authors study the problem of geographical forwarding in lossy areas where transitional regions are met, thus resulting in bad link quality and deterioration of transmission capabilities. In [5] a geographical routing protocol which exploits recently discovered paths to reduce the latency of the discovery process is proposed. This protocol shows significant performance improvement over traditional geographical routing protocols.

Similar approaches for performing cross-layer geographical routing have been proposed also in [17] and [4] with the aim of both reducing energy consumption and the forwarding overhead. In [17] nodes are assumed to turn ON and $\mathrm{OFF}$ as in energy limited scenarios. Accordingly, the next relay node is not known a priori, due to the nodes' duty cycle. Consequently, upon need of forwarding a data packet, the current source node sends a message which contains both its own coordinates and the coordinates of the destination. Then, a receiver contention scheme takes place and, ideally, the best available relay node is chosen based on geometrical considerations. In particular, the relay node is chosen to be the closest node towards the destination among the available slices in which the considered forwarding area is assumed to be subdivided. This solution, even if simple, does not guarantee data delivery since the sender node could not find any awake relay node in its proximity when forwarding data. Moreover this solution does not take into account the possibility of transmitting with different power levels.

The present paper extends the idea proposed in [4]. There, an integrated MAC-Routing protocol, called MACRO, which exploits the capability of tuning the transmission power of the sensor devices, is introduced. MACRO does not require any location information to be exchanged in the network and, thus, reduces energy consumption. In fact, the proposed protocol requires that each node only knows its own position and the position of the destination and forwarding is done according to geographical criteria. More specifically, in order to select the next relay node in the forwarding process, a competition is triggered at each hop in such a way that the most energy efficient choice is taken. Holes in the network can be also avoided through use of a control policy which allows to support reliable delivery of data also in case of low connectivity networks.

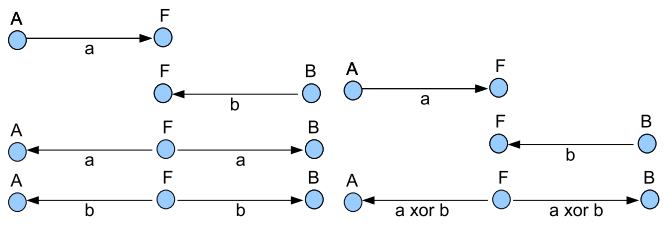

(a)

(b)

Figure 1: Network coding example.

\subsection{Network coding}

Network coding is an exciting and relatively recent field in information theory [1]. The idea underlying network coding can be illustrated using the three nodes scenario shown in Figure 1. Suppose that nodes $A$ and $B$ want to exchange packets through an intermediate node $F$. With the traditional method, node $A$ (resp. $B$ ) sends a packet $a$ (resp. $b$ ) to $F$, and then $F$ sends packets $a$ and $b$ in sequence, as shown in Figure 1(a). By using network coding, instead, node $F$ may recombine the input packets into one. In particular, node $F$ broadcast $a$ XOR $b$ instead of $a$ and $b$ in sequence. Both nodes $A$ and $B$ can recover the packets of interest reducing at the same time the number of transmissions (see Figure 1(b)). If more packets have to be combined, the XOR operation is replaced by a linear combination of the packets.

A primary result of network coding is that it increases the capacity and energy efficiency of the network [3]. Moreover, robustness is another benefit. In fact, by combining packets, network coding leads to equally important encoded packets [6]. So the receiver, provided that it receives a sufficient number of encoded packets, is able to decode the original information. Several works have exploited the network coding strategy to improve the throughput of multi-hop wireless networks by taking into account the broadcast nature of the wireless medium [7]. An example is [12], where the authors propose COPE, a new architecture for wireless mesh networks employing network coding. The aim of the paper is to bridge theory with practice. To this end the authors evaluate their architecture on a 20-node wireless network testbed. Results obtained show a large increase in network throughput. A detailed analytical evaluation of COPE has been presented in [16].

Other works apply network coding to residential wireless mesh networks. In [11] the authors suppose that all the transmissions are broadcast and overheard by the neighbors. In this way more packets can be encoded and retransmitted. 
Several experiments show that in some cases this approach can increase the network throughput by a factor two.

\section{MACRO+}

In this section we describe the system under consideration and the MACRO+ algorithm.

\subsection{System description}

To describe the MACRO+ scheme we focus on the behavior of a node $A$ that at time $t=0$ listens a packet $P_{B D}$ relayed from node $B$ in its radio coverage, supposed to be constant and equal to $r$, and directed to node $D$. Note that $B$ may be either the source of the packet or an intermediate node. We assume that all packets have the same payload and that the header size is $S_{H}$ when network coding is not applied and $S_{H_{N C}}$ when network coding is applied. Obviously, when network coding is applied the packet header is larger, i.e., $S_{H_{N C}}>S_{H}$, more specifically, $S_{H_{N C}} \approx 2 \cdot S_{H}$.

We call $\sigma_{A}$ the node density in the proximity of node $A$. We assume that node density does not change significantly in the surrounding region of each node. We also assume that each node is equipped with location computation facilities, e.g., GPS cards, and, therefore, can evaluate its current position. Furthermore, each node forwarding a packet inserts into it's header information about its own position and the position of the final destination, if not already included. Accordingly, $A$ is aware of the pairs of coordinates $\left(x_{A}, y_{A}\right)$, $\left(x_{B}, y_{B}\right)$, and $\left(x_{D}, y_{D}\right)$, i.e., its position and the positions of $B$ and the final destination $D$, respectively ${ }^{1}$.

Let $U, V$, and $W$ be three generic points of coordinates $\left(x_{U}, y_{U}\right),\left(x_{V}, y_{V}\right)$, and $\left(x_{W}, y_{W}\right)$. In the following we denote the angle formed by the arrays connecting $U$ to $V$ and $V$ to $W$ as $\theta_{U V W}$, whereas we denote the distance between any two points $U$ and $V$ as $d_{U, V}$.

At time $t=0$ it is possible that node $A$ has already a certain number of packets $N_{P}$ waiting to be relayed. Network coding can be utilized to merge the packet $P_{B D}$ with one of the other $N_{P}$ packets, thus exploiting a unique transmission.

\subsection{Scheme overview}

At any time a given node $A$ maintains updated a list containing information about the $N$ packets scheduled for transmission. More specifically, for any $i \leq N$ the list contains the entire packet, $P_{i}$, the time instance $t_{i}$ when such packet has been received, the time $\tau_{i}$ when the transmission of $P_{i}$ is scheduled, and a flag $\phi_{i}$ that is set equal to one if the packet will be transmitted encoded with another packet according to linear network coding. If this is the case, then $A$ also maintains a reference, $l_{i}$, to the packet that will be encoded together with $P_{i}$, and the value of the time instant when the transmission of the encoded packet is scheduled, $\tau_{i}^{*}$.

We assume that linear network coding is applied to pairs of packets. More specifically, the network encoder input is a pair of packets, whereas its output is one packet in which the header result from the concatenation of the headers of the two input packets, and the payload is the results of the XOR between the payloads of the two input packets.

At a generic time instant $t$ four different events may occur, as described in the following sections.

\footnotetext{
${ }^{1}$ Here we are assuming a two-dimensional space; however, extension to a three-dimensional space is straightforward.
}

\section{Event 1: A new packet is received.}

Suppose that the packet $P$ has been transmitted by node $B$ and is directed to node $D$. As soon as $A$ listens the packet it evaluates how good it is to transmit it without network coding. This goodness is quantified by means of a parameter which we call expected progress without network coding per unit of energy, $\rho$. Formal definition and analytical derivation of $\rho$ will be given in Section 3.3.1. Then for each $i \leq N$, if the $i-t h$ packet in the list, $P_{i}$, is not planned to be encoded, i.e., $\phi_{i}=0$, then $A$ evaluates how good it is to transmit the result of linear network coding between the current packet $P$ and $P_{i}$. This goodness is quantified by means of a parameter which we call expected progress with network coding per unit of energy, $\rho_{i}$. Formal definition and analytical derivation of $\rho_{i}$ will be given in Section 3.3.2. Let $\rho^{*}$ be defined as the highest value of $\rho_{i}$, i.e.

$$
\rho^{*}=\max _{\forall i \leq N: \phi_{i}=0}\left\{\rho_{i}\right\}
$$

and $j$ is the corresponding packet entry, i.e. $\rho_{j}=\rho^{*}$. Then, the new packet is inserted in the list in position $N+1$, the value $t_{N+1}$ is set to the current time ${ }^{2}$, whereas the packet transmission is scheduled at $\tau_{N+1}$ given by:

$$
\tau_{N+1}=t_{N+1}+\Delta t
$$

where $\Delta t$ is a random value generated according to an exponential distribution with average value reciprocal to the value $\rho$. Observe that, in this way, the higher is $\rho$, the earliest is the transmission scheduled. Better relay nodes try to relay a packet earlier than others.

Obviously, if $\rho \geq \rho^{*}$, then $\phi_{N+1}$ is set equal to zero. Instead, if $\rho<\rho^{*}$ then $\phi_{N+1}$ is set to one, $l_{N+1}$ is set equal to $j$ and $\tau_{N+1}^{*}$ is set as follows:

$$
\tau_{N+1}^{*}=t_{N+1}+\Delta \tau
$$

where $\Delta \tau$ is again a random value generated according to an exponential distribution with average value reciprocal to $\rho^{*}$. Clearly, also the $j$-th line of the list must be updated. More specifically, $\phi_{j}$ is set equal to one, and $l_{j}$ is set equal to $N+1$. Instead the transmission of the coded packet is scheduled to $\tau_{j}^{*}=t_{j}+\Delta \tau$. If $\tau_{j}^{*}$ is already elapsed, i.e., $\tau_{j}^{*}<t_{N+1}$, then, the transmission is scheduled to an instant coming very shortly, i.e., $\tau_{j}^{*}=t_{N+1}+\delta \tau$, where $\delta \tau$ is a short random value, i.e., the following relationship typically holds $\delta \tau<<\Delta \tau$.

\section{Event 2: A scheduled transmission time occurs.}

Suppose that the time of a scheduled non-coded transmission occurs, i.e., $t=\tau_{i}$ (we will consider the case $t=\tau_{i}^{*}$ later). In this case, node $A$ broadcasts a short message to notify it will take the responsibility of the transmission of packet $P_{i}$. We denote such message as $R T S_{i}$. Note that $R T S_{i}$ must only contain the information required to identify $P_{i}$. Then the node $A$ waits for a confirmation of sending the message, denoted as $C T S_{i}$, sent from the last relay of packet $P_{i}$, which we call $B_{i}$. If $C T S_{i}$ is not received for a certain time interval, $t_{R T O}$, then a new transmission of packet $P_{i}$ is scheduled at time $t=t_{R T O}+\tau_{i}-t_{i}$.

If at time $t$ the transmission of a coded packet has been scheduled, i.e., $t=\tau_{i}^{*}$, then $A$ broadcasts a request to send message which contains information about packet $P_{i}$ and

\footnotetext{
${ }^{2}$ We are neglecting the processing time which is very low.
} 
the packet that must be coded with it, i.e., $P_{l_{i}}$. We call such message $R C S_{i \oplus l_{i}}$. Then $A$ waits for the consequent confirmation to send messages $C T S_{i}$ and $C T S_{l_{i}}$ by nodes $B_{i}$ and $B_{l_{i}}$. If such messages are not received within a certain timeout, then new transmissions of packets $P_{i}$ and $P_{l_{i}}$ will be scheduled at times $\tau_{i}$ and $\tau_{l_{i}}$, respectively. This will be obtained by simply setting $\phi_{i}$ and $\phi_{l_{i}}$ to zero.

\section{Event 3: An RTS is received.}

Node $A$ controls whether it hears an RTS related to a packet that it has forwarded. If this is the case, it generates an appropriate CTS, then waits to hear the transmission of the actual packet. When such transmission occurs, node $A$ is not responsible for the packet anymore and therefore deletes the corresponding entry from its list.

Obviously, if the RTS is related to a packet that node $A$ has not transmitted, then $A$ neglects it.

\section{Event 4: A CTS is received.}

If a CTS is received we must distinguish two different cases. If the CTS is the response to an RTS transmitted by node $A$, then node $A$ transmit the related packet and reschedules its transmission after an appropriate timeout, called $t_{R T O_{1}}$. If the CTS is the response to the RTS transmitted by some other node, then node $A$ searches in its list of packet transmission schedules. Suppose that the entry corresponding to the received CTS occupies the $j$-th entry in the list. If node $A$ was planning to transmit such packet using linear encoding, i.e., $\phi_{j}=1$, then the entry of the packet $l_{j}$ must be updated. More specifically, $\phi_{l_{j}}$ is set equal to zero. Then the $j$-th entry is deleted and the list updated accordingly.

\subsection{Expected progress per unit of energy}

In this section we formally define the expected progress parameters. More specifically, in Section 3.3.1 we analyze the case where network coding is not applied, whereas in Section 3.3.2 we consider the case where network coding is applied.

\subsubsection{Expected progress without network coding per unit of energy}

In evaluating the expected progress towards the destination, node $A$ needs to compare the expected distance between the next best relay and the destination before and after it forwards the packets.

More specifically, let us call

- $R_{(B, D)}$ the best next relay, with the exclusion of node $A$, for a packet forwarded by node $B$ towards the destination $D$. In other words $R_{(B, D)}$ represents the node in the radio coverage of $B$, with the exclusion of $A$, which is the nearest to the destination $D$.

- $R_{(A, D)}$ the best next relay for a packet forwarded by node $A$ towards the destination $D$. This represents the node in the radio coverage of $A$ which is the nearest to the destination $D$.

Note that node $A$ does not know the positions of $R_{(B, D)}$ and $R_{(A, D)}$. Therefore, in its perspective the distances between $D$ and $R_{(B, D)}$ as well as $R_{(A, D)}$ are random variables that we call $\Delta_{R_{(B, D)}, D}$ and $\Delta_{R_{(A, D)}, D}$, respectively. Accordingly, the expected progress of the packet towards the destination $D$ achieved thanks to $A$ 's forwarding is given by

$$
\gamma=E\left\{\Delta_{R_{(B, D)}, D}-\Delta_{R_{(A, D)}, D}\right\}
$$

Accordingly, the expected progress without network coding per unit of energy, $\rho$, is the ratio between $\gamma$ and the energy spent to transmit a packet, i.e.,

$$
\rho=\frac{\gamma}{\left(S_{H}+S_{D}\right) \cdot \epsilon_{b}}
$$

where $\epsilon_{b}$ is the energy per bit and $S_{H}$ and $S_{D}$ are the packet header and payload length in bit, respectively. Substituting eq. (4) in eq. (5) and observing that $E\{\cdot\}$ is a linear operator, we can write

$$
\rho=\left[E\left\{\Delta_{R_{(B, D)}, D}\right\}-E\left\{\Delta_{R_{(A, D)}, D}\right\}\right] \cdot \frac{1}{\left(S_{H}+S_{D}\right) \cdot \epsilon_{b}}
$$

In the following we calculate $E\left\{\Delta_{R_{(B, D)}, D}\right\}$; derivation of $E\left\{\Delta_{R_{(A, D)}, D}\right\}$ is identical.

By definition $E\left\{\Delta_{R_{(B, D)}, D}\right\}$ can be computed as:

$$
E\left\{\Delta_{R_{(B, D)}, D}\right\}=\int_{-\infty}^{+\infty} x \cdot f_{\Delta}(x) d x
$$

where $f_{\Delta}(x)$ represents the probability density function of the random variable $\Delta_{R_{(B, D)}, D}$.

The function $f_{\Delta}(x)$ can be calculated as the derivative of the corresponding probability distribution function $F_{\Delta}(x)$ which is defined as follows:

$$
F_{\Delta}(x)=\operatorname{Pr}\left\{\Delta_{R_{(B, D)}, D} \leq x\right\}
$$

being $0 \leq x<r$.

The probability in the right hand side of eq. (8) is equal to the probability that the node within the coverage area of $B$, which is the closest to the destination $D$, is distant from $D$ at least $(d-x)$. This means there should be no nodes in the outlined area shown in Figure 2 and called $a(d-x, d)$, representing the area of intersection between two circles, namely the one centered in $B$ and having radius equal to $r$ and the other centered in $D$ with radius equal to $(d-x)$ and $0 \leq x<r$.

If we assume that nodes are Poisson-distributed in the sensed area with density $\lambda=N / A$, where $A$ represents the area of the entire sensed region, it follows that the probability distribution function in eq. (8) can be calculated as

$$
F_{\Delta}(x)= \begin{cases}0 & x<0 \\ e^{-\lambda \cdot a(d-x, d)} & 0 \leq x<r \\ 1 & x \geq r\end{cases}
$$

Accordingly, eq. (7) can be rewritten as follows:

$$
\begin{aligned}
& E\left\{\Delta_{R_{(B, D)}, D}\right\}=\int_{-\infty}^{+\infty} x \cdot f_{\Delta}(x) d x \\
& =\left[x \cdot F_{\Delta}(x)\right]_{0}^{r}-\int_{0}^{r} F_{\Delta}(x) d x= \\
& =r-\int_{0}^{r} F_{\Delta}(x) d x
\end{aligned}
$$

In order to solve eq. (10) we need to calculate the area $a(d-x, d)$. For sake of simplicity, we define $y=d-x$. Accordingly:

$$
a(y, d)=\Psi+\Omega-\Phi
$$


where $\Psi, \Omega$, and $\Phi$ can be calculated applying Euclidean geometry as described in [8]. The geometrical meaning of $\Psi, \Omega$ and $\Phi$ can be clarified looking at Figure 2 (a). In this figure $\Psi$ is the circular sector $\mathrm{BKH}, \Omega$ is the circular sector DKH and $\Phi$ is the polygon BKDH.

Now we can evaluate the expected progress $E\left\{\Delta_{R_{(B, D)}, D}\right\}$ as given in eq. (10) where the integral in the right hand side can be easily calculated numerically.

\subsubsection{Expected progress with network coding per unit of energy}

Suppose that node $A$ performs linear coding of two packets $P_{1}$ and $P_{2}$ that it has received by $B_{1}$ and $B_{2}$ and that must be delivered to final destinations $D_{1}$ and $D_{2}$ respectively. Let us call $P_{1 \oplus 2}$ the packet resulting from encoding the two packets.

Let us define $\gamma_{1}$ the expected progress of packet $P_{1}$ towards the destination $D_{1}$ that can be achieved through a transmission by $A$, and $\gamma_{2}$ the analogous for packet $P_{2}$.

The expected progress when applying network coding, per unit of energy, $\rho_{x}$ is defined as:

$$
\rho_{x}=\frac{\left(\gamma_{1}+\gamma_{2}\right) \cdot 1}{\left(S_{H_{N C}}+S_{D}\right) \cdot \epsilon_{b}}
$$

In the following we derive how to calculate $\gamma_{1}$ while derivation of $\gamma_{2}$ is identical.

Observe that if node $A$ encodes packets $P_{1}$ and $P_{2}$ in a single packet $P_{1 \oplus 2}$, then only nodes that have received both $P_{1 \oplus 2}$ and $P_{2}$ can decode $P_{1}$. In other words, $P_{1}$ can be decoded only by nodes in the radio coverage of both $A$ and $B_{2}$. Accordingly, let $R_{\left(A, B_{2}, D_{1}\right)}$ be the best relay for packet $P_{1}$ following $A$ towards the destination $D_{1}$ in the radio coverage of both $A$ and $B_{2}$. Moreover, analogously to the definition provided in Section 3.3.1, let $R_{\left(B_{1}, D_{1}\right)}$ be the best next relay of packet $P_{1}$ following a transmission by $B_{1}$. In other words, $R_{\left(B_{1}, D_{1}\right)}$ represents the node in the radio coverage of $B_{1}$ which is nearest to the destination $D_{1}$. Observe that $A$ is not aware of the positions of $R_{\left(A, B_{2}, D_{1}\right)}$ and $R_{\left(B_{1}, D_{1}\right)}$. Therefore, in node $A$ 's perspective the distances between $D_{1}$ and $R_{\left(A, B_{2}, D_{1}\right)}$ as well as $R_{\left(B_{1}, D\right)}$ are random variables. We call such variables $\Delta_{R_{\left(A, B_{2}, D_{1}\right)}, D_{1}}$ and $\Delta_{R_{\left(B_{1}, D_{1}\right)}, D_{1}}$.

According to the above definitions, we can calculate $\gamma_{1}$ as

$$
\begin{aligned}
\gamma_{1} & =E\left\{\Delta_{R_{\left(B_{1}, D_{1}\right)}, D_{1}}-\Delta_{R_{\left(A, B_{2}, D_{1}\right)}, D_{1}}\right\}= \\
& =E\left\{\Delta_{R_{\left(B_{1}, D_{1}\right)}, D_{1}}\right\}-E\left\{\Delta_{R_{\left(A, B_{2}, D_{1}\right)}, D_{1}}\right\}
\end{aligned}
$$

Note that the term $E\left\{\Delta_{R_{\left(B_{1}, D_{1}\right)}, D}\right\}$ can be calculated as explained in Section 3.3.1. For what concerns the term $E\left\{\Delta_{R_{\left(A, B_{2}, D_{1}\right)}, D_{1}}\right\}$, its derivation can be performed by estimating the area $a(d-x, d)$ used in eq. (10). This area can be estimated considering the intersection between the coverage area of node $A$, the coverage area of node $B_{2}$ and the direction of the destination $D_{1}$. To better clarify this aspect let us refer to Figure 2 (b).

More specifically, we should evaluate the area $a(d-x, d)$ of the circular triangle KHL. This area can be derived using the basics of geometry as the sum of the area of the circular sector ALH, the circular sector $B_{2} \mathrm{KH}$ and the circular sector $D_{1} \mathrm{KL}$ summed to the area of the triangle KHL. Finally, $E\left\{\Delta_{R_{\left(A, B_{2}, D_{1}\right)}, D_{1}}\right\}$ can be estimated.

\section{NUMERICAL RESULTS}

In this section, we show the energy efficiency increase which can be obtained by combining the advantages of network coding with geographical routing. To this purpose we will compare the expected progress per unit of energy when network coding is used and when it is not, in the case that $\epsilon_{b}=1, S_{H}=20$ Byte, $S_{D}=256$ Byte and $S_{H_{N C}}=40$ Byte. More in details we will consider three different cases.

The first scenario refers to the condition where two nodes $B_{1}$ and $B_{2}$, the one opposite to the other, have some packets to transmit to their destinations $D_{1}$ and $D_{2}$, respectively. A forwarder node $A$ is located in between nodes $B_{1}$ and $B_{2}$, as shown in Figure 3 (a). This is the scenario where applying network coding results useful. In fact, as can be seen from Figure 3 (b), the expected progress with network coding per unit of energy, $\rho_{x}$, vs. nodes' density, results greater with respect to the case when no network coding is employed, $\rho_{1}$ and $\rho_{2}$. This is because the two traffic flows follow opposite directions and thus the area $a(d-x, d)$, defined in Section 3.3 .2 , results maximized. The advantage of networking coding results almost double for high values of nodes' density because more possible better relays can be found.

The second scenario, shown in Figure 4(a), refers to the intermediate condition where applying network coding is useful but the maximization is reduced with respect to the previous case, as evident looking at Figure 4(b). This is because now the two nodes $B_{1}$ and $B_{2}$ are not located perfectly opposite with respect to $A$ and thus the area $a(d-x, d)$ is not maximized. This implies that the expected number of nodes which can be used to perform advantageously network coding is reduced with respect to Figure 3 . Note that in this case, the values of $\rho_{1}$ and $\rho_{2}$ are different because the areas, related to $B_{1}$ and $B_{2}$, where the relay nodes should be found are different in size.

Finally, the third scenario is shown in Figure 5 (a). Here we observe that the nodes which should allow a progress of the encoded packet $P_{1 \oplus 2}$ towards the destinations $D_{1}$ and $D_{2}$, respectively, would be located further away from node A's location. This would imply that a decrease in the expected progress with network coding per unit of energy can be observed with respect to the case when no network coding is considered. This is evident looking at figure 5 (b).

\section{CONCLUSIONS}

In this paper we have introduced MACRO+: a geographical routing scheme able to exploit network coding. According to MACRO+ nodes receiving a packet evaluate their goodness to forward the packet towards the destination and schedule a tentative to assume the responsibility for the forwarding after a time interval that depends on such goodness. More specifically, the better the node the earlier the tentative. In this paper we have described MACRO+ and evaluated its advantages in a set of scenarios through numerical examples.

We are currently working to address the following issues:

- The proposed scheme assumes that the location of the destination is known. However, methods are required to allow mobile terminals to learn the location of the destination of the information they generate. In this context the idea of homezone proposed in [9] could be applied.

- Mobile terminals will be placed in areas where network 


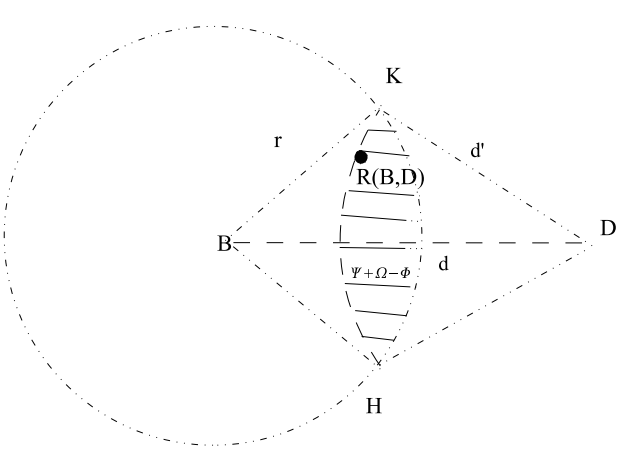

(a) Area where the next hop must be found in case network coding is not used.

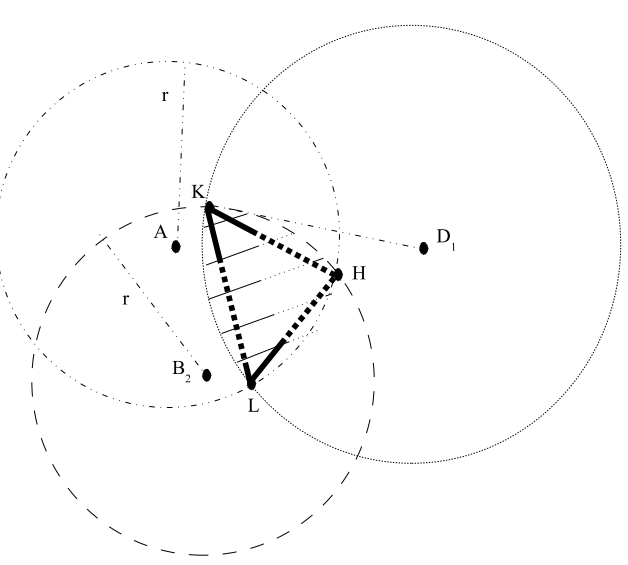

(b) Area where the next hop must be found in case network coding is used.

Figure 2: Areas where the next hop must be found.

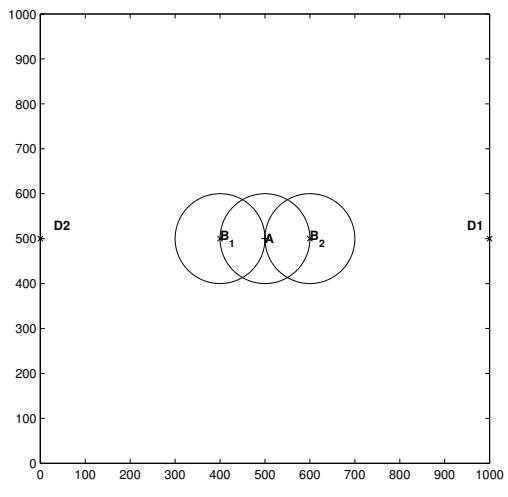

(a)

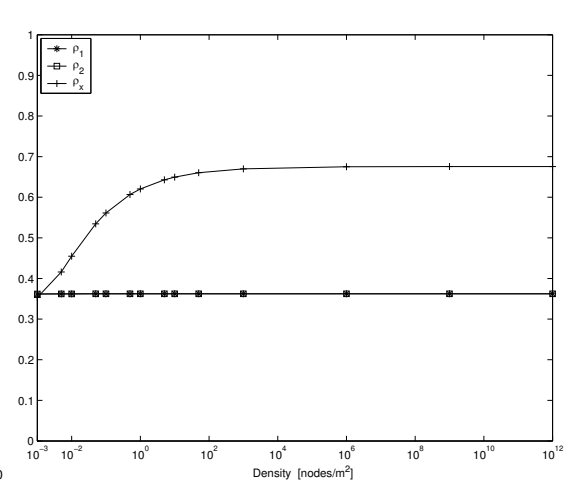

(b)

Figure 3: First scenario (a), and expected progress per unit of energy (b).

infrastrucutere is likely to be available. Such infrastructure could be used to solve problems in the geographical routing algorithm caused by the presence of so called network holes in the ad hoc network. Appropriate algorithms are required to allow such exploitation.

\section{REFERENCES}

[1] R. Ahlswede, N. Cai, S.-Y. R. Li, and R. W. Yeung. Network information flow. IEEE Transactions on Information Theory, 46(4), July 2000.

[2] Android. In www.openhandsetalliance.com.

[3] C. Chekuri, C. Fragouli, and E. Soljanin. On average throughput and alphabet size in network coding. IEEE Transactions on Information Theory, 52(6), January 2006.

[4] D. Ferrara, L. Galluccio, A. Leonardi, G. Morabito, and S. Palazzo. Macro: An integrated mac/routing protocol for geographical forwarding in wireless sensor networks. In Infocom, Miami, FL, USA, March 2005.

[5] S. Fotopoulou-Prigipa and B. McDonald. Gcrp: Geographic virtual circuit routing protocol for ad hoc networks. In $M A S S$, Fort Lauderdale, FL, USA, October 2004.

[6] C. Fragouli, J.-Y. L. Boudec, and J. Widmer. Network coding: an instant primer. ACM SIGCOMM Computer Communication Review, 36(1), January 2006.

[7] C. Fragouli, J. Widmer, and J.-Y. L. Boudec. A network coding approach to energy efficiency broadcasting: from theory to practice. In IEEE Infocom, Barcelona, Spain, April 2006.

[8] L. Galluccio, A. Leonardi, G. Morabito, and S. Palazzo. A trade-off between energy consumption reduction and responsiveness in information delivery for delay-tolerant sensor networks with mobile sink. In 


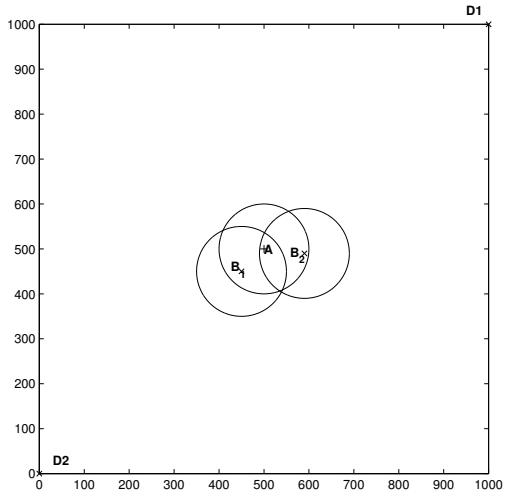

(a)

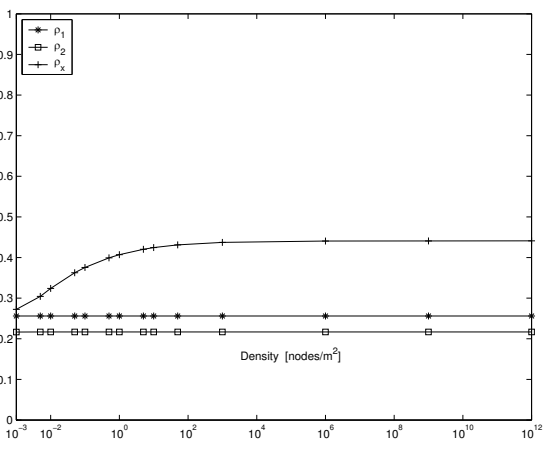

(b)

Figure 4: Second scenario (a), and expected progress per unit of energy (b).

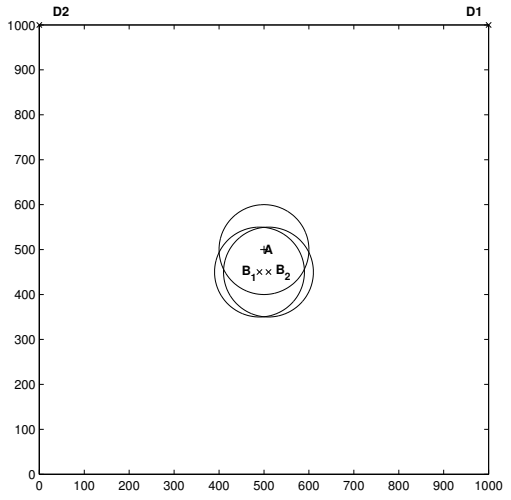

(a)

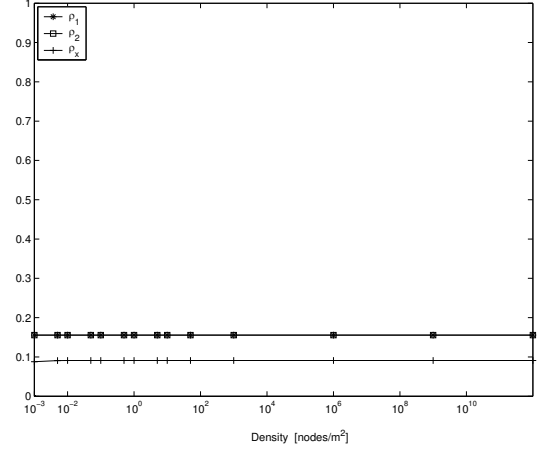

(b)

Figure 5: Third scenario (a), and expected progress per unit of energy (b).

ACM IWCMC, Vancouver, BC, Canada, July 2006.

[9] S. Giordano and M. Hamdi. Mobility management: The virtual home region. Tech. Rep., October 1999.

[10] B. Karp and H. T. Kung. Gpsr: Greedy perimeter stateless routing for wireless networks. In International Conference on Mobile Computing and Networking (MobiCom), Boston, MA, USA, August 2000.

[11] S. Katti, D. Katabi, W. Hu, H. S. Rahul, and M. Medard. The importance of being opportunistic: Practical network coding for wireless environment. In Allerton Annual Conference on Communication, Control and Computing, Monticello, IL, USA, September 2005.

[12] S. Katti, H. Rahul, W. Hu, D. Katabi, M. Medard, and J. Crowcroft. Xors in the air: Practical wireless network coding. In ACM SIGCOMM, Pisa, Italy, September 2006.

[13] M. J. Lee, J. Zheng, X. Hu, H. hui Juan, C. Zhu, Y. Liu, J. S. Yoon, and T. Saadawi. A new taxonomy of routing algorithms for wireless mobile ad hoc networks: the component approach. IEEE

Communications Magazine, 44(11), November 2006.

[14] A. Rao, S. Ratnasamy, C. Papadimitriou, S. Shenker, and I. Stoica. Geographic routing without location information. In International Conference on Mobile Computing and Networking (MobiCom), San Diego, CA, USA, September 2003.

[15] K. Seada, M. Zuniga, A. Helmy, and B. Krishnamachari. Energy-efficient forwarding strategies for geographic routing in lossy wireless sensor networks. In ACM Sensys, Baltimore, MD, USA, November 2004.

[16] S. Sengupta, S. Rayanchu, and S. Banerjee. An analysis of wireless network coding for unicast sessions: The case for coding-aware routing. In IEEE Infocom, Anchorage, Alaska, USA, May 2007.

[17] M. Zorzi and R. Rao. Geographic random forwarding (geraf) for ad hoc and sensor networks: Multihop performance. IEEE/ACM Transactions on Mobile Computing, 2(4), Oct.-Dec. 2003. 